\title{
La situación de la salud-enfermedad-atención de la primera infancia en la Ciudad de Buenos Aires entre 1990 y 2002
}

\author{
The situation of health-disease-care \\ in early childhood in the City of \\ Buenos Aires between 1990 and 2002
}

Victoria Mazzeo ${ }^{1}$

\footnotetext{
${ }^{1}$ Lic. en Sociología, Universidad de Buenos Aires. Doctora en Ciencias Sociales, FLACSO Sede Argentina. Responsable de la Unidad de Análisis Demográfico, Dirección General de Estadística y Censos, Gobierno de la Ciudad de Buenos Aires. vmazzeo@buenosaires.gov.ar
}

RESUMEN A partir de la década del '90 se presenta un proceso de creciente desigualdad en la sociedad, con sectores que registran mayores dificultades en el acceso a bienes y servicios, ya sea por sus bajos ingresos o por la pérdida de sus derechos sociales como consecuencia de la precarización laboral. En este contexto, los niños representan el grupo de mayor vulnerabilidad en donde los condicionantes sociales, económicos, culturales y ambientales son determinantes de su situación de salud.

El propósito del artículo fue describir, a partir de datos secundarios, los cambios en la mortalidad de la primera infancia y en la atención de la salud infantil de la Ciudad de Buenos Aires, para el período 1990-2002, diferenciando grupos sociales y/o áreas espaciales.

PALABRAS CLAVE Proceso Salud-Enfermedad; Sector de Atención de Salud; Morbilidad; Mortalidad infantil.

ABSTRACT Starting the 1990 s a process of increasing inequality impinges on society
with sectors that, as a result of labour precarization, register higher difficulties to have
access to goods and services either due to lower income or to loss of their social rights.
In this context children represent the group of higher vulnerability where social, economic,
cultural and environmental conditions are determinant factors of their health situation.
Therefore, the purpose of this article was to describe, for the 1990-2002 period, using
secondary data, the changes in early childhood mortality and the child health care of the
City, differentiating social groups and/or spatial areas.

KEY WORDS Health-Disease Process; Health Care Sector; Morbidity; Infant Mortality. 
Las condiciones de salud de una población son el resultado del efecto conjunto de factores genéticos, estilos de vida y factores del contexto, donde desarrolla su vida. Es decir, la situación de la salud y sus desigualdades están determinadas tanto individual como histórica y socialmente. Si bien los factores genéticos pueden tener un peso relevante en la salud individual, a nivel de la población, son los factores socioeconómicos, culturales, ambientales y políticos los que tienen el rol fundamental como determinantes de los niveles de salud. Es así, que los cambios económicos y sociales promovidos en la década del '90 -de debilitamiento del Estado y hegemonía del mercado en sociedades que ya registraban situaciones de desigualdad- profundizaron los déficits de los sectores que registraban mayores dificultades en el acceso a bienes y servicios por sus bajos ingresos o por la pérdida de sus derechos sociales como consecuencia de la precarización laboral. Este cambio afectó más a las familias pobres, que son las que tienen más niños, quienes resultan especialmente perjudicados porque son objeto de una crianza que inhibe la posibilidad de un desarrollo sano en los primeros años de vida y puede malograr el resto de su existencia.

Cuando una sociedad se empobrece es previsible un empeoramiento de las condiciones de salud de sus integrantes. ¿Cómo explicar entonces el descenso experimentado por la mortalidad de la primera infancia ante el incremento de la pobreza y la desigualdad socioeconómica? Esta constatación lleva a reflexionar sobre:

a. cuáles son las causas de la reducción de la mortalidad de la primera infancia;

b. quiénes fueron los niños que se beneficiaron de esa mejora.

\section{LOS CAMBIOS EN LA COBERTURA DE SALUD}

El mercado de trabajo de la Ciudad de Buenos Aires fue afectado por las políticas económicas aplicadas en el país durante los años '90. Ellas generaron un fuerte impacto sobre las condiciones de vida de la población, especialmente sobre la fuerza de trabajo, que se vio afectada por la pérdida de puestos estables, por el incremento de la desocupación, la subocupación horaria, la precariedad laboral y la disminución del promedio de horas semanales trabajadas. Asimismo, en los últimos años se produjo una estratificación en el acceso a los servicios de salud que se relaciona con los niveles socioeconómicos de la población. Los sectores de mayores ingresos están en condiciones de pagar medicina privada directamente o contratando seguros. Los asalariados registrados son atendidos por un sistema de seguridad social cuya atención depende de la calidad de las obras sociales, muy diferentes entre sí, que más de una vez incluyen prestaciones deficientes. Por último, los grupos de menores recursos no tienen otra opción que acudir a un deteriorado sistema público de salud. Esta constatación lleva a reflexionar sobre la existencia de disparidades en la cobertura de salud y, por ende, en la atención de la salud de los distintos sectores sociales; específicamente en el grupo más vulnerable que son los niños.

Con el objeto de verificar esta afirmación y confirmar la mayor desprotección de la primera infancia (a) en la prevención y atención de su salud, se analizó lo ocurrido en la ciudad, tomando como indicadores la cobertura de salud y la condición de pobreza medida por el método de las necesidades básicas insatisfechas (NBI) a partir del procesamiento de los datos de los dos últimos censos de población (b), asumiendo que aquellos sin cobertura explícita podían utilizar los servicios públicos.

La evidencia empírica muestra (ver Cuadro 1) que, tanto para la población pobre como para la no pobre, se incrementó la proporción de aquellos que carecen de una cobertura explícita, (entre los primeros el $41 \%$ y entre los segundos el $36 \%$ ). Es decir, de aquellos que no tienen protección de la seguridad social (obra social) y/o seguros privados. Es indudable que esta situación es mucho más grave entre los pobres cuya participación en la cobertura del sistema público triplica a la de los no pobres. Esta desprotección es aún más evidente en los niños, y entre ellos, los pobres, para quienes los niveles de cobertura del sistema público resultaban superiores al 50\% en 1991 y llegaron a superar el $70 \%$ en 2001. Los valores obtenidos verifican para los pobres y no pobres, la reducción de la proporción con obra social y la importante caída 
CUadro 1. POBLACIÓN EN HOGARES PARTICULARES POR CONDICIÓN DE NBI Y TIPO DE COBERTURA EN SALUD. POBLACIÓN TOTAL Y PRIMERA INFANCIA. CIUDAD DE BUENOS AIRES, 1991 Y 2001.

\begin{tabular}{|c|c|c|c|c|}
\hline \multirow[t]{2}{*}{ CONDICIÓN NBI } & \multicolumn{2}{|c|}{$\mathrm{NO} N B \mathrm{I}$} & \multicolumn{2}{|c|}{$\mathrm{NBI}$} \\
\hline & 1991 & 2001 & 1991 & 2001 \\
\hline \multicolumn{5}{|l|}{ COBERTURA DE SALUD } \\
\hline \multirow[t]{2}{*}{ Población total (1) } & 100,0 & 100,0 & 100,0 & 100,0 \\
\hline & $(2.619 .669)$ & $(2.512 .999)$ & $(228.452)$ & $(212.489)$ \\
\hline Tiene sólo obra social (2) & 52,6 & 47,3 & 43,7 & 30,8 \\
\hline Tiene sólo plan médico o mutual & 13,1 & 20,3 & 2,8 & 2,8 \\
\hline Tiene obra social y plan médico o mutual & 17,4 & 9,5 & 7,5 & 1,7 \\
\hline No tiene obra social ni plan médico o mutual & 16,9 & 22,9 & 46,0 & 64,7 \\
\hline \multirow[t]{2}{*}{ Población de 0 a 4 años (1) } & 100,0 & 100,0 & 100,0 & 100,0 \\
\hline & $(147.412)$ & 131.515 & $(26.824)$ & $(24.319)$ \\
\hline Tiene sólo obra social (2) & 47,4 & 43,9 & 37,2 & 24,8 \\
\hline Tiene sólo plan médico o mutual & 15,7 & 21,6 & 2,3 & 1,7 \\
\hline Tiene obra social y plan médico o mutual & 18,6 & 9,3 & 4,8 & 0,9 \\
\hline No tiene obra social ni plan médico o mutual & 18,3 & 25,2 & 55,7 & 72,6 \\
\hline \multicolumn{5}{|c|}{$\begin{array}{l}\text { Notas: } \\
\text { (1)Para } 1991 \text { se descontaron los ignorados de tipo de cobertura. } \\
\text { (2)Para } 1991 \text { se sumó los que tenían obra social e ignoraban plan médico o mutual. }\end{array}$} \\
\hline
\end{tabular}

de la población con obra social y plan médico o mutual. Se destaca que estas disminuciones afectaron con mayor intensidad a los pobres para quienes la cobertura de obra social se redujo un $30 \%$ (pasó del 44 al 31\%), mientras que entre los no pobres lo hizo en un $11 \%$ (pasó del 53 al $47 \%$ ).

Evidentemente, los cambios en las relaciones de trabajo y la precariedad laboral afectaron profundamente las posibilidades de una inserción estable. Esto provocó, para un número importante de familias, la pérdida de la obra social y les generó la dependencia de un sistema público colapsado. Esta pérdida afectó a los niños en dos aspectos inherentes a su salud: la prevención y la atención. Para acceder en el servicio público a los beneficios que brinda el sistema de seguridad social, las familias sin seguro de salud necesitan probar su condición de pobreza y pasar por los inconvenientes de trámites burocráticos y largas esperas para el otorgamiento de turnos.

En síntesis, el incremento de la desocupación abierta, la subocupación horaria y la precariedad laboral, así como la caída de los ingresos familiares per capita en un contexto de agravamiento de su distribución desigual, configuran una situación de pobreza de capacidades y de medios y cercenan las posibilidades de asegurar a los niños un crecimiento pleno de todas sus potencialidades.

\section{LA SITUACIÓN DE LA SALUD-ENFERMEDAD-ATENCIÓN}

Desde hace varios años, pero especialmente durante la última década, estudios teóricos 
y empíricos demostraron significativas diferencias en las condiciones de salud entre diferentes grupos sociales definidos de acuerdo con su nivel de ingresos, educación, origen étnico, género, área de residencia o por factores ambientales $(1,2)$. Ahora bien, si la desigualdad de las relaciones sociales afecta y compromete tanto a los pobres -favoreciendo la falta de cohesión social- también impacta profundamente en la situación de la infancia.

Con el objeto de caracterizar la situación de la salud-enfermedad-atención de la primera infancia entre 1990 y 2002, se seleccionaron algunos indicadores capaces de reflejar la evolución de la morbimortalidad infantil. Debido a las limitaciones existentes en las fuentes de datos para el análisis de la morbilidad, se utilizó la importancia relativa de los diagnósticos de los egresos de los niños menores de cinco años residentes en la ciudad, que en los años 1995 y 2000 estuvieron internados en los hospitales dependientes del Gobierno de la Ciudad de Buenos Aires. Si bien se trata de una información parcial, limitada al sector oficial provincial y a los diagnósticos más graves que requirieron internación, permite un conocimiento aproximado de los problemas de salud-enfermedad-atención de la población carenciada.

Asimismo, a partir de las estadísticas vitales de ese período, se analizaron indicadores considerados de riesgo al nacimiento y para la sobrevivencia de la primera infancia: madres con baja instrucción, solas, adolescentes o de alta paridez y de los nacimientos de pretérmino o de bajo peso en el total de nacimientos de madres residentes en la ciudad. ¿Por qué estos indicadores? Es sabido que el nivel educativo de la madre condiciona el grado de seguimiento de conductas recomendadas y está fuertemente asociado a la sobrevivencia de los niños (2,3). Los embarazos de madres adolescentes son considerados de alto riesgo debido a que cuanto más joven es la madre mayor es el riesgo de morbimortalidad por complicaciones del embarazo, parto y puerperio (4). También existen potenciales efectos para la salud de los hijos quienes quedan expuestos a nacer con bajo peso (5). Las consecuencias económicas y sociales de la fecundidad temprana reducen eventualmente las posibilidades educativas de las madres, lo que incide en su calidad de vida y en la de sus hijos $(6,7)$. La prematurez y el peso al nacer son considerados predictores de las probabilidades de sobreviviencia de los niños durante el primer mes de vida (8).

También se analizaron las tasas de mortalidad de la primera infancia según grupo de edad y causa de muerte. Es ampliamente conocida la asociación entre mortalidad de la primera infancia y las condiciones de vida (9-15). En este caso, al igual que con los indicadores de riesgo al nacimiento, la fuente de información fueron las estadísticas vitales del período 1990-2002 referidas a los niños residentes en la Ciudad de Buenos Aires.

\section{LOS CAMBIOS EXPERIMENTADOS EN LA MORBILIDAD}

Para el análisis de la morbilidad de la población existen dos fuentes de información: los egresos hospitalarios y las consultas externas que se realizan en los establecimientos de cada jurisdicción. Lamentablemente, el Programa Nacional de Estadísticas de Salud sólo centraliza la información del subsector oficial. Lo mismo ocurre en el Gobierno de la Ciudad de Buenos Aires: el Ministerio de Salud sólo maneja la información de los hospitales que dependen de ella. Esta situación pone en evidencia el sesgo de cobertura que se suma a otro de los problemas que dificultaron el análisis. Para el período analizado, no existe información sobre los diagnósticos de las consultas externas según residencia habitual del paciente, sólo hay datos para el total de consultas por especialidad y establecimiento. Por otro lado, los diagnósticos principales de los informes estadísticos de hospitalización se codificaron en la ciudad únicamente en los años terminados en 0 y 5, pero para 1990 no hay datos y en 1995 la codificación fue realizada por el Programa Nacional de Estadísticas Vitales a través de una muestra probabilística. Cuando se obtuvieron las bases de egresos hospitalarios de los años 1995 y 2000, se detectaron, además, problemas referidos a la falta de codificación del domicilio dentro de la ciudad y a la ausencia de las variables socioeconómicas que no permitió investigar la distribución espacial de la morbilidad ni sus diferenciales socioeconómicos. De esta manera, 
Cuadro 2. PRIMERA INFANCIA. ESTRUCTURA DE LOS EGRESOS HOSPITALARIOS SEGÚN PRINCIPALES DIAGNÓSTICOS POR GRUPO DE EDAD. CIUDAD DE BUENOS AIRES, 1995.

\begin{tabular}{lccc}
\hline DIAGNÓSTICO DE EGRESO & $\begin{array}{c}\text { MENORES DE } 1 \\
\text { ANNO }\end{array}$ & $\begin{array}{c}\text { 1 A 4 AÑOS } \\
\text { TOTAL PRIMERA } \\
\text { INFANCIA }\end{array}$ \\
\hline Total & 100,0 & 100,0 & 100,0 \\
Duración corta de la gestación y bajo peso al nacer & $(1.083)$ & $(438)$ & $(1.521)$ \\
Ictericia perinatal & 15,0 & 0,0 & 10,7 \\
Neumonía no especificada & 14,8 & 0,0 & 10,5 \\
Crecimiento fetal lento y desnutrición fetal & 4,7 & 11,6 & 6,7 \\
Bronquiolitis aguda & 8,1 & 0,0 & 5,8 \\
Infección intestinal & 6,5 & 1,6 & 5,1 \\
Traumatismo intracraneal & 2,6 & 8,0 & 4,1 \\
Hipoxia intrauterina y asfixia al nacer & 0,9 & 8,2 & 3,0 \\
Otras afecciones respiratorias del feto y del recién nacido & 4,1 & 0,0 & 2,9 \\
Quemaduras de sitios múltiples especificados & 4,0 & 0,0 & 2,8 \\
Resto enfermedades respiratorias & 0,4 & 7,3 & 2,4 \\
Resto traumatismos y envenenamientos & 1,9 & 7,1 & 3,4 \\
Resto causas & 0,9 & 11,2 & 3,9
\end{tabular}

Fuente: Elaboración propia (1) en base a estadísticas de egresos hospitalarios de la Ciudad de Buenos Aires. Dirección de Estadísticas e Información de Salud, Ministerio de Salud, Presidencia de la Nación.

sólo fue posible conocer el perfil epidemiológico de la morbilidad de los niños residentes en la Ciudad de Buenos Aires que estuvieron internados en esos dos años en los hospitales dependientes del gobierno local.

El eje del análisis se orientó a conocer el diagnóstico de egreso del episodio de internación según la edad del paciente. Para ello se examinaron, en primer lugar, las categorías diagnósticas agrupadas por capítulo de la clasificación de enfermedades, y luego, las principales causas de morbilidad a tres dígitos (c).

Al tener en cuenta los diagnósticos más frecuentes se observa que en 1995 (ver Cuadro 2) la corta duración de la gestación, el bajo peso al nacer y la ictericia perinatal representaron casi la tercera parte de los egresos de los menores de un año, mientras que, esa misma proporción, para los egresos de los niños de 1 a 4 años fue por neumonía, infección intestinal, traumatismo intracraneal y quemaduras múltiples.
Para el año 2000 (ver Cuadro 3), la estructura de los diagnósticos de los egresos hospitalarios se modificó: continuaron siendo prioritarios los egresos referidos al período perinatal $(35 \%)$, pero aumentaron su importancia relativa los egresos por enfermedades respiratorias $(22 \%)$. Es lógico pensar entonces, que los factores socioeconómicos, de accesibilidad a servicios de salud y nutricionales, sumados a las condiciones ambientales, hayan incidido en este incremento.

Si se comparan las estructuras de los diagnósticos de egresos de ambos años, con la cautela que amerita haber trabajado con dos revisiones diferentes, se observa que la participación de los niños que quedaron internados se redujo, por tratarse de nacimientos en pretérmino o con bajo peso (diagnósticos típicos del grupo neonatal precoz), mientras que se incrementó la proporción de las infecciones respiratorias, enfermedades diarreicas y accidentes 
(más frecuentes en el grupo posneonatal y de 1 a 4 años), que deberían resolverse en el primer nivel de atención.

Esto no significa que haya existido una mejora en la proporción de nacimientos con bajo peso -por el contrario, según las estadísticas vitales del período, ésta aumentó el 7\% entre 1995 y 2002- sino que se incrementaron notoriamente las afecciones respiratorias. Cabe destacar que, según la Organización Mundial de la Salud, las infecciones respiratorias agudas constituyen una de las principales causas de consulta en los servicios de salud de América Latina. Los niños de la región padecen entre cuatro y seis episodios por año (16).

En lo que respecta al control prenatal, el hecho de que la gran mayoría de las mujeres asista a los controles prenatales, y que casi la totalidad de los partos sean institucionales, hace evidente que el contacto de las mujeres con los servicios de salud no garantiza una calidad adecuada en la atención y un cuidado preventivo ante los eventuales riesgos asociados al embarazo y al parto (17). Lamentablemente, no existen datos sobre el estado nutricional y de salud de las mujeres en edad fértil; y por lo tanto, no es posible establecer el riesgo del niño de nacer con bajo peso para su edad gestacional como consecuencia del déficit nutricional sufrido por su madre desde niña. Sólo se cuenta con datos sobre la proporción de los nacimientos de bajo peso al nacer o de pretérmino, que en la ciudad fueron del 6,7 y $5,6 \%$ respectivamente, en los inicios de la década del '90 -probablemente

Cuadro 3. PRIMERA INFANCIA. ESTRUCTURA DE LOS EGRESOS HOSPITALARIOS SEGÚN PRINCIPALES DIAGNÓSTICOS POR GRUPO DE EDAD. CIUDAD DE BUENOS AIRES, 2000.

\begin{tabular}{|c|c|c|c|}
\hline DIAGNÓSTICO DE EGRESO & $\begin{array}{l}\text { MENORES DE } 1 \\
\text { AÑO }\end{array}$ & 1 A 4 AÑOS & $\begin{array}{l}\text { TOTAL PRIMERA } \\
\text { INFANCIA }\end{array}$ \\
\hline \multirow[t]{2}{*}{ Total } & 100,0 & 100,0 & 100,0 \\
\hline & (6.791) & (3.022) & (9.813) \\
\hline Bronquitis aguda & 13,2 & 5,5 & 10,8 \\
\hline Neumonía no especificada & 3,6 & 9,4 & 5,4 \\
\hline $\begin{array}{l}\text { Transtornos relacionados con duración corta de la gestación y } \\
\text { con bajo peso al nacer }\end{array}$ & 8,0 & 0,0 & 5,5 \\
\hline $\begin{array}{l}\text { Transtornos relacionados con embarazo prolongado y con } \\
\text { sobrepeso al nacer }\end{array}$ & 7,6 & 0,0 & 5,2 \\
\hline Ictericia neonatal por otras causas & 6,6 & 0,0 & 4,6 \\
\hline Diarrea y gastroenteritis aguda & 3,0 & 8,0 & 4,6 \\
\hline Dificultad respiratoria del recién nacido & 5,4 & 0,0 & 3,7 \\
\hline Traumatismo intracraneal & 1,3 & 6,4 & 2,9 \\
\hline Retardo de crecimiento fetal y desnutrición fetal & 3,8 & 0,0 & 2,6 \\
\hline $\begin{array}{l}\text { Feto y recién nacido afectados por otras complicaciones del } \\
\text { trabajo de parto y del parto }\end{array}$ & 3,3 & 0,0 & 2,3 \\
\hline Resto enfermedades respiratorias & 5,1 & 15,0 & 8,1 \\
\hline Resto traumatismos y envenenamientos & 2,1 & 13,2 & 5,5 \\
\hline Resto causas & 37,0 & 42,5 & 38,7 \\
\hline
\end{tabular}

Fuente: Elaboración propia (1) en base a estadísticas de egresos hospitalarios de la Ciudad de Buenos Aires. Dirección de Estadísticas e Información de Salud, Ministerio de Salud, Presidencia de la Nación. 
asociados a la crisis económica- y se incrementaron al 7,4 y $6,6 \%$ respectivamente al comenzar la década siguiente. Ambas condiciones determinan disparidades en las oportunidades de sobrevivir, crecer y desarrollarse normalmente. No obstante los esfuerzos mayores que requieran por parte de su familia y de la sociedad, estos niños verán afectada su capacidad de desarrollo pleno (6-8).

Debe tenerse en cuenta que los países con mayor desarrollo y con mejor organización y equidad en salud, como por ejemplo Dinamarca y Suecia, presentan participaciones del bajo peso al nacer cercanas al $4 \%$ (18). Este porcentaje indica la brecha que existe, en este indicador de calidad de vida, entre la situación en la Ciudad de Buenos Aires y el paradigma representado por los países con mayores niveles de salud del mundo. El potencial de reducción es del $42 \%$ sobre la base de una incidencia de bajo peso al nacer del 7,3\% en la Ciudad de Buenos Aires para 2002.

Otra asociación entre la salud infantil y las variables sociales es la que existe entre el nivel de instrucción de la madre y las posibilidades de sobrevivencia y crianza de los niños. Se comprobó que el grado de seguimiento de las conductas recomendadas está condicionado por el nivel educativo de la madre $(6,7,16)$, y que éste es un instrumento fundamental para asegurar, a través de estrategias de promoción, mejores condiciones de salud para la infancia. Según las estadísticas vitales del período analizado en la Ciudad de Buenos Aires, la incidencia de nacimientos de madres con baja instrucción (d), en ambas décadas, representó menos del 3\%. La mayor escolarización de las mujeres en esta ciudad explica la diferencia con los valores del promedio nacional $(16,6 \%$ en $1991-1992$ y $10,1 \%$ en 2001-2002). En el extremo opuesto se encuentran Santiago del Estero con el 48,2\% en el bienio 1991-1992 y Misiones, con el 33,2\% en el bienio 2001-2002.

Otro de los riesgos al nacimiento lo constituye el embarazo adolescente. Las adolescentes no tienen el mismo acceso a los métodos de planificación familiar, y se exponen a tener embarazos no deseados y a verse forzadas al uso de métodos de control de la natalidad muy traumáticos y peligrosos, como el aborto inducido, que derivan en mayor riesgo de mortalidad. En Buenos Aires, la maternidad adolescente se mantuvo estable: representó cerca del $6 \%$ de los nacimientos, muy por debajo del promedio nacional (14,7\% en $1991-1992$ y $14,6 \%$ en $2001-2002)$.

Los indicadores analizados permiten afirmar, que a pesar de la crisis, la Ciudad de Buenos Aires continúa manteniendo una posición de privilegio con respecto al resto del país.

\section{LOS CAMBIOS EN LA MORTALIDAD}

Es ampliamente conocida la asociación entre mortalidad infantil y condiciones de vida $(7,11,14,19,20)$. Las tasas de mortalidad infantil, especialmente de menores de cinco años, se convirtieron no sólo en indicadores de salud, sino también de bienestar y desarrollo humano (6), ya que reflejan la distribución y disponibilidad de los servicios de saneamiento, salud y educación de la población. Si bien históricamente la Ciudad de Buenos Aires registra los menores niveles de mortalidad del país, se consideró conveniente, dada su heterogeneidad socioeconómica, incorporar el análisis de la mortalidad de la primera infancia como indicador de la "no salud" de la misma.

El nivel de la mortalidad de la primera infancia descendió el 40\% entre 1990 y 2002. Si se tienen en cuenta los distintos grupos etarios en que ésta puede segmentarse se observa que, partiendo de distintos niveles, las mayores reducciones se produjeron entre los niños de 1 a 4 años y en la mortalidad neonatal (ver Cuadro 4).

También se consideró necesario investigar cuáles fueron las causas de muerte ya que éstas son consideradas la expresión biológica final de un proceso que en una proporción relevante está determinado por la estructura económica y social de una sociedad. A través de su análisis, quedarían evidenciadas las formas particulares de enfermar y morir, de cada conjunto social, que responden a condiciones de vida específicas.

La estructura por causas de la mortalidad de la la primera infancia se analizó para tres grupos etarios: neonatal, posneonatal y de 1 a 4 años, porque la importancia relativa de las causas difiere según la edad (ver Cuadro 5). En la mortalidad neonatal los mayores niveles se registraron 
Cuadro 4. TASA DE MORTALIDAD PERINATAL Y DE LA PRIMERA INFANCIA SEGÚN GRUPO DE EDAD (POR MIL NACIDOS VIVOS). CIUDAD DE BUENOS AIRES, 1990-2002.

\begin{tabular}{|c|c|c|c|c|c|}
\hline AÑO & PERINATAL & NEONATAL & POSTNEONATAL TOTAL INFANTIL & 1 A 4 AÑOS & $\begin{array}{l}\text { PRIMERA } \\
\text { INFANCIA }\end{array}$ \\
\hline
\end{tabular}

$\begin{array}{lllllll}1990 & 13,9 & 10,7 & 5,3 & 16,0 & 2,9 & 18,9 \\ 1991 & 12,6 & 8,5 & 5,1 & 13,6 & 2,2 & 15,7 \\ 1992 & 13,6 & 10,2 & 4,2 & 14,4 & 2,2 & 16,6 \\ 1993 & 12,5 & 10,4 & 5,0 & 15,4 & 2,3 & 17,7 \\ 1994 & 12,9 & 9,6 & 4,4 & 14,0 & 1,0 & 14,9 \\ 1995 & 10,8 & 8,3 & 4,2 & 12,5 & 2,8 & 15,3 \\ 1996 & 11,6 & 9,6 & 4,7 & 14,3 & 2,2 & 16,5 \\ 1997 & 8,1 & 3,7 & 11,8 & 2,0 & 13,8 \\ 1998 & 10,8 & 8,6 & 4,0 & 12,6 & 1,7 & 14,3 \\ 1999 & 10,9 & 7,1 & 3,2 & 10,3 & 1,6 & 11,9 \\ 2000 & 9,5 & 5,9 & 3,1 & 9,0 & 1,2 & 10,2 \\ 2001 & 7.8 & 6,2 & 2,9 & 9,1 & 1,3 & 10,4 \\ 2002 & 7,4 & 6,3 & 3,6 & 9,9 & 1,4 & 11,3\end{array}$

Fuente: Elaboración propia (1) en base a estadísticas vitales de nacimientos y defunciones de menores de un año de residentes en la Ciudad de Buenos Aires. Período 1990-2002. Dirección General de Estadística y Censos, Gobierno de la Ciudad de Buenos Aires.

en "bajo peso al nacer", "anomalías congénitas" y en "causas de la mortalidad perinatal (excluido bajo peso al nacer)". La tendencia de la mortalidad por bajo peso al nacer, está relacionada con el aumento de la proporción de nacimientos de bajo peso. Durante la última década se incrementó especialmente la participación de los nacidos con menos de 1.000 gramos, que son los de más difícil sobrevivencia, pese a que la tecnología neonatal logró avances importantes mediante mejoras en el equipamiento para el tratamiento y recuperación de los recién nacidos, y también por las intervenciones terapéuticas adecuadas (e). Por otro lado, la estructura de la tasa de mortalidad infantil según peso al nacer, revela que los niños nacidos con bajo peso (menos de 2.500 gramos) representan más del $60 \%$ de la tasa de mortalidad infantil total (1). Esta participación es muy alta comparada con la registrada en 1990 en los países del oeste europeo, donde constituían cerca del $30 \%$ de la tasa de mortalidad infantil total (21).

En la mortalidad posneonatal (Cuadro 5) los mayores niveles se ubicaron en las "enfermedades del aparato respiratorio" y en "anomalías congénitas". Finalmente, la mortalidad de los niños de 1 a 4 años presentó una mayor heterogeneidad en su estructura por causas, siendo las más importantes los "accidentes", las "enfermedades infecciosas y parasitarias", las "malformaciones congénitas del corazón", la "neumonía e influenza" y las "enfermedades que afectan al mecanismo de la inmunidad" (incluye SIDA). En el último año se destaca un ligero repunte de la mortalidad por enfermedades infecciosas y parasitarias, causas relacionadas con el medio físico y social, la malnutrición y la falta de higiene, todas ellas asociadas con contextos socioeconómicos desfavorables.

Es lógico pensar que los factores socioeconómicos -que condicionan la accesibilidad a los servicios de salud y la situación nutricional- hayan podido incidir en el aumento de la participación de formas graves de infecciones respiratorias. Los factores de riesgo que determinan la gravedad del proceso son el bajo peso al nacer, la edad, el lento desarrollo del mecanismo inmunológico y la contaminación atmosférica en general (16). También los accidentes, una de las principales causas de muerte entre los niños de 1 
Cuadro 5. TASAS DE MORTALIDAD POR GRUPO DE EDAD SEGÚN CAUSAS DE MUERTE (POR DIEZ MIL). CIUDAD DE BUENOS AIRES, 1990-2002.

\begin{tabular}{|c|c|c|c|c|c|c|c|c|c|c|c|c|c|c|c|c|c|c|c|}
\hline \multirow[b]{2}{*}{ AÑO } & \multicolumn{6}{|c|}{ MORTALIDAD NEONATAL } & \multicolumn{6}{|c|}{ MORTALIDAD POSNEONATAL } & \multicolumn{7}{|c|}{ MORTALIDAD 1 A 4 AÑOS } \\
\hline & (a) & (b) & (c) & (d) & (e) & Total & (f) & (g) & (c) & (d) & (e) & Total & (h) & (i) & (j) & (k) & (l) & (d) & Total \\
\hline 1990 & 18,1 & 57,6 & 23,0 & 5,3 & 3,9 & 107,9 & 7,0 & 4,4 & 9,4 & 22,5 & 9,2 & 52,5 & 2,2 & 4,8 & 2,4 & 1,9 & 1,2 & 16,2 & 28,8 \\
\hline 1991 & 12,4 & 46,8 & 17,1 & 4,1 & 4,7 & 85,1 & 5,2 & 3,5 & 7,7 & 21,0 & 13,1 & 50,5 & 1,2 & 2,5 & 2,7 & 1,7 & 1,0 & 13,1 & 22,2 \\
\hline 1992 & 24,8 & 43,1 & 19,6 & 5,8 & 8,3 & 101,5 & 4,4 & 3,1 & 8,8 & 17,3 & 8,5 & 42,1 & 1,5 & 4,4 & 0,5 & 1,8 & 1,0 & 12,9 & 22,2 \\
\hline 1993 & 26,0 & 40,8 & 27,3 & 6,7 & 3,1 & 103,9 & 9,1 & 2,6 & 14,8 & 17,2 & 6,8 & 50,4 & 2,3 & 4,4 & 0,3 & 1,8 & 1,8 & 12,2 & 22,9 \\
\hline 994 & 28,8 & 40,0 & 20,4 & 3,9 & 3,1 & 96,2 & 4,8 & 2,0 & 12,0 & 18,5 & 6,1 & 43,4 & 0,8 & 2,0 & 0,5 & 0,5 & 1,0 & 4,8 & 9,7 \\
\hline 1995 & 25,8 & 30,8 & 18,4 & 3,4 & 4,5 & 83,0 & 8,2 & 2,7 & 6,5 & 17,4 & 7,5 & 42,2 & 2,5 & 2,2 & 1,2 & 3,0 & 2,5 & 16,2 & 27,6 \\
\hline 996 & 28,8 & 34,0 & 26,5 & 4,7 & 2,5 & 96,5 & 9,0 & 3,3 & 12,8 & 16,7 & 5,0 & 46,8 & 2,8 & 2,5 & 1,5 & 0,3 & 2,0 & 12,5 & 21,5 \\
\hline 1997 & 24,0 & 31,5 & 19,2 & 4,4 & 2,0 & 81,1 & 7,7 & 2,6 & 9,5 & 14,7 & 2,3 & 36,8 & 1,3 & 5,4 & 1,3 & 1,8 & 1,8 & 8,4 & 19,9 \\
\hline 1998 & 28,3 & 30,3 & 21,8 & 5,0 & 1,3 & 86,7 & 12,6 & 2,3 & 11,4 & 12,1 & 1,3 & 39,7 & 0,8 & 1,5 & 0,8 & 1,5 & 2,0 & 10,1 & 16,7 \\
\hline 1999 & 26,8 & 21,5 & 19,0 & 3,6 & 0,5 & 71,4 & 11,2 & 1,5 & 7,5 & 10,2 & 1,5 & 31,9 & 2,0 & 4,1 & 0,2 & 0,7 & 1,7 & 7,6 & 16,3 \\
\hline 2000 & 24,3 & 14,7 & 14,5 & 5,2 & 0,5 & 59,2 & 11,0 & 1,6 & 7,3 & 10,0 & 1,1 & 31,0 & 2,1 & 3,0 & 0,7 & 0,5 & 0,2 & 5,5 & 11,9 \\
\hline 2001 & 24,7 & 14,7 & 19,2 & 2,8 & 0,7 & 62,1 & 8,1 & 0,5 & 8,8 & 10,6 & 1,4 & 29,4 & 0,7 & 3,1 & 0,9 & 0,2 & 0,0 & 7,8 & 12,8 \\
\hline 2002 & 23,3 & 14,5 & 19,6 & 2,4 & 3,2 & 63,0 & 12,0 & 1,7 & 10,3 & 9,6 & 2,4 & 36,0 & 0,2 & 2,7 & 1,2 & 0,5 & 0,7 & 8,6 & 14,0 \\
\hline
\end{tabular}

Notas:

(a) Bajo peso al nacer

(b) Perinatales (excluido bajo peso al nacer)

(c) Anomalías congénitas

d) Resto bien definidas

(e) Desconocidas y mal definidas

(f) Enfermedades del aparato respiratorio

(g) Septicemia-Meningitis

(h) Malformaciones congénitas del corazón

(i) Causas externas

(j) Infecciones parasitarias

(k) Neumonía e influenza

(I) Mecanismo de la inmunidad

Fuente: Elaboración propia (1) en base a estadísticas vitales de nacimientos y defunciones de menores de un año de residentes en la Ciudad de Buenos Aires. Período 1990-2002. Dirección General de Estadística y Censos, Gobierno de la Ciudad de Buenos Aires.

a 4 años, constituyen eventos prevenibles. Los factores de riesgo de accidentes se relacionan con las características del ambiente y su entorno inmediato. Se debe tener presente que el niño debe ser considerado junto con su entorno familiar. La evidencia empírica muestra que las caídas constituyen el tipo más frecuente de accidente, mientras que las quemaduras son más habituales en los niveles socioeconómicos bajos, donde pueden incidir el hacinamiento y las fuentes de luz y calor sin protección (16).

La medida del grado de atención de la salud está en relación no sólo con los resultados que se obtienen (morbilidad y mortalidad), sino también con el riesgo de la población que se asiste. Un indicador utilizado para analizar la calidad de la atención de la salud en los niños menores de un año es el índice obtenido al dividir la tasa de mortalidad infantil, elegida como resultado, por la proporción de nacimientos con bajo peso al nacer, seleccionada como riesgo de la población (22). Para los niños de 1 a 4 años se utiliza un índice diferente, ya que el bajo peso deja de ser un factor de tan alto riesgo como sucede durante el primer año de vida. En este caso, el índice utiliza la tasa de mortalidad por muertes no evitables (tumores, anomalías congénitas y cardiovasculares) dividida por la tasa de mortalidad por muertes evitables (infecciosas, violencias y respiratorias). Ambas relaciones muestran que cuanto mayor es el índice, peor sería la atención de la salud. 
Figura 1. EVALUACIÓN DE LA ATENCIÓN DE LA SALUD DE LA PRIMERA INFANCIA. CIUDAD DE BUENOS AIRES, 1990-2002.

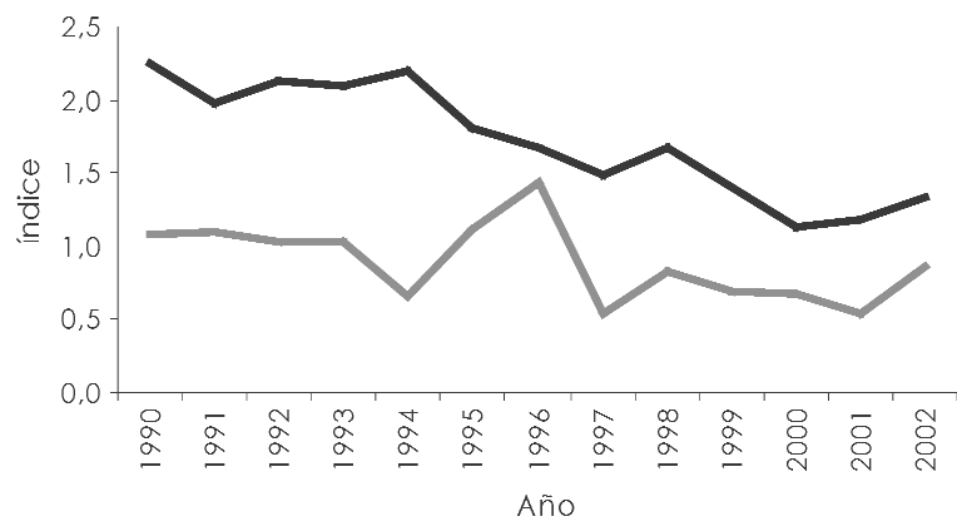

-1 año 1-4 años

Fuente: Elaboración propia (1) en base a estadísticas vitales de nacimientos y defunciones de menores de un año de residentes en la Ciudad de Buenos Aires. Período 1990-2002. Dirección General de Estadística y Censos, Gobierno de la Ciudad de Buenos Aires.

En la Ciudad de Buenos Aires, en ambos grupos de edad se observa una reducción del índice (Figura 1), si bien con algunas fluctuaciones, muestra una mejora en la atención desde mediados de los años '90, aunque se registra un deterioro en el último bienio. Se destaca que durante todo el período fue peor la calidad de la atención de los menores de un año. Las explicaciones a esta situación se encuentran en las conductas de las mujeres en el control efectivo de sus embarazos. La ausencia de controles o su reducida cantidad se vincula con el bajo peso al nacer, los nacimientos prematuros y la mortalidad infantil, indicadores incorporados en el índice elaborado (1).

Es posible suponer que la crisis económica que empujó a la mujer hacia el mercado laboral para aportar ingresos a los deteriorados presupuestos familiares, modificó las prácticas cotidianas en el cuidado de los niños. Probablemente tuvo menos tiempo para compartir con sus hijos y para llevarlos a los controles periódicos y/o menores ingresos para transportarlos hasta el hospital o consultorio médico. También incidirían en la calidad del cuidado el estrés y el cansancio y la menor chance de lactancia.

\section{LA DESIGUALDAD EN LA SALUD-ENFERMEDAD}

Las dificultades para traducir y operacionalizar los conceptos de los diversos abordajes teóricos sobre el proceso salud-enfermedad en estudios empíricos con datos secundarios, han Ilevado a los investigadores ha utilizar aproximaciones para explicar la causalidad de las desigualdades. En este caso se consideró la residencia habitual de la madre como "proxy" del sector social de pertenencia del hogar, ya que lamentablemente las estadísticas vitales, por la ausencia de preguntas adecuadas, no permiten elaborar indicadores por condición socioeconómica.

La desigualdad en la salud-enfermedad de los niños se midió aplicando los Coeficientes de Gini y de Concentración a los indicadores seleccionados para conocer el riesgo al nacimiento y la mortalidad según residencia habitual de la madre ( $f$ ). Es sabido que cuanto mayor es el área comprendida entre la Curva de Lorenz y la diagonal de igualdad, mayor será el valor del Coeficiente de Gini y por lo tanto la desigualdad 
de la distribución del indicador que se está midiendo. Por su parte, una Curva de Concentración (g) cercana a la de Lorenz, indicaría que las zonas con peores indicadores de salud, serían aproximadamente las mismas que las que presentan peores indicadores socioeconómicos.

A comienzos de la década del '90 (ver

Cuadro 6) los indicadores que presentaron mayor desigualdad en los riesgos al nacimiento fueron: madres con baja instrucción, adolescentes y de alta paridez. Por otro lado, se destaca el bajo nivel de los coeficientes bajo peso al nacer y pretérmino que, a pesar de lo esperado, prácticamente están cercanos a la diagonal de igualdad. Varios autores (23-28) observaron que el bajo peso al nacer y el pretérmino no son sensibles para analizar desigualdades en salud ni estarían asociados con los ingresos, debido a que el alto porcentaje de nacimientos por cesárea (h) ocultaría las diferencias socioeconómicas de ambos indicadores. Es decir, las frecuentes cesáreas en las clases media y alta, "compensarían" el efecto de la desnutrición o falta de controles adecuados de las mujeres de la clase baja. También existen causas biológicas que se relacionan con los nacimientos de pretérmino o con el retardo del crecimiento intrauterino, entre otras: aborto espontáneo, contractilidad uterina aumentada, acortamiento del cuello uterino, malformaciones uterinas, diabetes con vasculopatía, placenta previa, rotura prematura de las membranas, embarazo múltiple y alta paridez (18).

En lo referido a las relaciones entre alta paridez y bajo peso al nacer, según las estadísticas vitales del período, se observa para la Ciudad de Buenos Aires que las participaciones del bajo peso fueron, durante todo el período analizado, más importantes entre las de alta paridez. Este hecho también está relacionado con la pobreza ya que las madres de alta paridez (i) en su mayoría pertenecen a los estratos bajos de la población (1). Con respecto a los indicadores de mortalidad, los mayores coeficientes se registraron en la mortalidad infantil por causas reducibles y en la posneonatal.

Cuadro 6. MEDICIÓN DE LA DESIGUALDAD EN LA SALUD Y LA MORTALIDAD DE LA PRIMERA INFANCIA. CIUDAD DE BUENOS AIRES, 1991-1992 Y 2001-2002.

\begin{tabular}{|c|c|c|c|c|}
\hline \multirow[b]{2}{*}{ INDICADOR } & \multicolumn{2}{|c|}{ 1991-1992 } & \multicolumn{2}{|c|}{$2001-2002$} \\
\hline & $\begin{array}{c}\text { COEFICIENTE } \\
\text { DE GINI }\end{array}$ & $\begin{array}{l}\text { COEFICIENTE DE } \\
\text { CONCENTRACIÓN }\end{array}$ & $\begin{array}{c}\text { COEFICIENTE } \\
\text { DE GINI }\end{array}$ & $\begin{array}{l}\text { COEFICIENT } \\
\text { CONCENTRA }\end{array}$ \\
\hline Madres con baja instrucción & 0,289 & $-0,265$ & 0,441 & $-0,375$ \\
\hline Madres solas & 0,175 & $-0,150$ & 0,207 & $-0,168$ \\
\hline Madres adolescentes & 0,231 & $-0,199$ & 0,280 & $-0,246$ \\
\hline Madres de alta paridez & 0,225 & $-0,178$ & 0,338 & $-0,309$ \\
\hline Nacimientos pretérmino & 0,064 & $-0,035$ & 0,056 & $-0,007$ \\
\hline Nacimientos bajo peso & 0,052 & $-0,029$ & 0,058 & $-0,039$ \\
\hline Nacimientos atendidos por médico & 0,160 & 0,111 & 0,204 & 0,170 \\
\hline Mortalidad fetal & 0,264 & $-0,166$ & 0,252 & $-0,153$ \\
\hline Mortalidad infantil & 0,141 & $-0,099$ & 0,207 & $-0,156$ \\
\hline Mortalidad infantil neonatal & 0,137 & $-0,071$ & 0,197 & $-0,129$ \\
\hline Mortalidad infantil posneonatal & 0,201 & $-0,157$ & 0,280 & $-0,207$ \\
\hline Mortalidad infantil reducible & 0,212 & $-0,118$ & 0,256 & $-0,197$ \\
\hline Mortalidad perinatal & 0,173 & $-0,104$ & 0,174 & $-0,123$ \\
\hline Mortalidad menores de 5 años & 0,126 & $-0,096$ & 0,202 & $-0,164$ \\
\hline
\end{tabular}

Fuente: Elaboración propia (1) en base a estadísticas vitales de nacimientos y defunciones de menores de un año de residentes en la Ciudad de Buenos Aires. Período 1990-2002. Dirección General de Estadística y Censos, Gobierno de la Ciudad de Buenos Aires. 
Cuadro 7. PARTICIPACIÓN DE LA POBLACIÓN QUE NO REALIZÓ CONSULTA MÉDICA EN LOS ÚLTIMOS SEIS MESES SEGÚN QUINTIL DE INGRESOS TOTAL FAMILIAR POR ZONA. CIUDAD DE BUENOS AIRES, 2002.

\begin{tabular}{lcc}
\hline ZONA & 20\% MÁS POBRE & 20\% MÁS RICO \\
\hline Total Ciudad & 37,3 & 26,4 \\
& $(120.727)$ & $(125.484)$ \\
Este & 34,2 & 25,5 \\
Norte & 31,4 & 23,5 \\
Oeste & 37,5 & 29,9 \\
Sur & 41,6 & 28,6
\end{tabular}

Fuente: Elaboración propia (1) en base a datos de la Encuesta Anual de Hogares de la Ciudad de Buenos Aires, 2002. Dirección General de Estadística y Censos, Gobierno de la Ciudad de Buenos Aires.

A comienzos de la década del 2000 se observa que en la mayoría de los indicadores (excepto pretérmino) se incrementó la desigualdad. No obstante, los que presentan mayores coeficientes continúan siendo los mismos que en la década anterior.

Los coeficientes obtenidos permitirían confirmar la existencia de una relación importante entre la evolución de la salud infantil y de la desigualdad social en la Ciudad de Buenos Aires. Se destaca que a partir de 2000 las diferencias entre los valores absolutos de ambos coeficientes (Gini y Concentración) se acortaron, lo que implica que las zonas con peores indicadores de salud son, aproximadamente las mismas que las que presentan peores niveles socioeconómicos. Los datos sugieren que la salud de los niños es altamente dependiente de las condiciones de vida en que se desarrolla. Indudablemente, la pertenencia a hogares con necesidades básicas insatisfechas es un factor que condicionó tanto las posibilidades de acceso a los servicios como las conductas en relación al proceso salud-enfermedad-atención de los niños.

Es sabido que la familia constituye la unidad donde, en forma directa o como mediadora, se establecen y operan algunos de los principales determinantes de la morbilidad y la mortalidad, en particular entre los menores de cinco años. Asimismo, es la unidad a partir de la cual se constituyen y/o se organizan las principales redes sociales respecto del proceso salud-enfermedad-atención (28). Algunos autores $(14,29)$ afirman que es la familia la que brinda al niño y a sus integrantes en general, los elementos para poder enfrentar condiciones adversas. De esta manera, favorecería o dificultaría en el niño las condiciones para un crecimiento y desarrollo sano.

Por tal motivo, se creyó conveniente incluir en el análisis de la desigualdad en la atención de la salud de las familias de la Ciudad de Buenos Aires, indicadores obtenidos a partir de la Encuesta Anual de Hogares 2002 (j) que permite calcular pobreza por ingresos según división territorial. A partir de la distribución de los hogares según quintiles de ingresos totales familiares se advierte que la no realización de una consulta médica en los seis meses anteriores a la entrevista, entre el 20\% más pobre y el $20 \%$ más rico (ver Cuadro 7), muestra comportamientos diferentes por zona (k). En el sur, la proporción de población del estrato más pobre que no consultó al médico es una vez y media la proporción del estrato más rico que no lo hizo. En el resto de la Ciudad de Buenos Aires las diferencias son menores.

Los resultados obtenidos indicarían que a pesar de la mayor concentración de centros de atención primaria en la zona sur, su población, y en especial la más pobre, tendría una percepción de la importancia de los controles médicos que la diferencia notablemente del resto de la ciudad. Por lo tanto, no es posible pensar que los problemas de la zona sur puedan resolverse con políticas focalizadas. En las zonas de mayor concentración de la pobreza son necesarias estrategias de intervención múltiple dada la heterogeneidad de problemas que sufre la población. 
En síntesis, los resultados obtenidos muestran que la creciente desigualdad socioeconómica incidió en las condiciones de salud de los niños de los hogares más pobres, especialmente con relación a los riesgos que pueden ser controlados a través de la prevención, diagnóstico y/o tratamiento oportuno, es decir, a través de la estrategia de la atención primaria. La Ciudad de Buenos Aires presenta una situación de profunda inequidad socioespacial. Haber trabajado a nivel de áreas menores puso al descubierto diferencias que deben ser abordadas para resolver las necesidades de los distintos espacios poblacionales.
La evidencia objetiva sobre las diferencias en las condiciones de salud y de vida representa el primer paso para distinguir las inequidades en salud (29). Este ejercicio descriptivo podría considerarse como un inicio en la identificación de las inequidades en salud de la primera infancia de la Ciudad de Buenos Aires. Resulta imperioso definir otro tipo de política pública más inclusiva y dirigida a integrar los sectores carenciados. Es necesario romper con el círculo perverso de la pobreza donde están envueltos los niños como prisioneros de su destino.

\section{NOTAS FINALES}

a. Definida como menores de cinco años.

b. Se utilizaron las bases del Censo Nacional de Población y Vivienda 1991 de la Ciudad de Buenos Aires y del Censo Nacional de Población, Hogares y Viviendas 2001 de la Ciudad de Buenos Aires, del Instituto Nacional de Estadística y Censos.

c. Se usaron dos clasificaciones de morbilidad: para 1995, la CIE $9^{\circ}$ Revisión, y para 2000, la CIE $10^{\circ}$ Revisión por lo cual fue necesario compatibilizarlas.

d. Hasta primario incompleto. e. Entre otras: administración prenatal de corticoides en amenaza de parto prematuro, administración precoz posdata de surfactante en prematuros, inducción de maduración intestinal con un programa de alimentación enteral precoz con leche humana en recién nacidos prematuros, lactancia materna.

f. Se seleccionó la Circunscripción Electoral por ser la división territorial utilizada para la elaboración de las estadísticas vitales y también censales.

g. La proporción de población con NBI fue el indicador utilizado para evidenciar los diferenciales socioeconómicos.

h. Según la Encuesta SIEMPRO 1997, en la Ciudad de Buenos Aires era el 32\% de los nacimientos de las mujeres que tenían hijos menores de 3 años. 
i. Definidas por el Programa Nacional de Estadísticas de Salud como las mujeres que tuvieron cinco hijos y más.

j. Encuesta que desde 2002 releva anualmente la Dirección General de Estadística y Censos del Gobierno de la Ciudad de Buenos Aires con el objeto de brindar información sobre la situación socioeconómica de la población de la ciudad a nivel territorial desagregado. El tamaño efectivo de la muestra fue de 8.500 hogares.

k. Este (Retiro, San Nicolás, Monserrat, San Telmo, Constitución, Balvanera, Almagro, Boedo y Caballito); Norte (Puerto Madero, Recoleta, Palermo, Colegiales, Belgrano y Nuñez); Oeste (Liniers, Mataderos, Flores, Floresta, Parque Avellaneda, Villa Luro, Velez Sarsfield, Versalles, Villa Real, Villa Devoto, Monte Casto, Villa del Parque, Villa Santa Rita, Villa Gral. Mitre, Paternal, Villa Crespo, Chacarita, Villa Ortúzar, Agronomía, Villa Pueyrredón, Villa Urquiza, Coghlan y Saavedra) y Sur (Boca, Barracas, San Cristóbal, Parque Patricios, Nueva Pompeya, Parque Chacabuco, Villa Soldati, Villa Lugano y Villa Riachuelo).

\section{REFERENCIAS BIBLIOGRÁFICAS}

1. Mazzeo V. La inequidad en la salud-enfermedad de la primera infancia. Las políticas de salud y la capacidad resolutiva de los servicios en la Ciudad de Buenos Aires. [Tesis de Doctorado en Ciencias Sociales]. Buenos Aires: FLACSO; 2006.

2. McQuestion MJ. Los comportamientos de salud correlacionados y la transición de la mortalidad en América Latina. Notas de Población. 2001;28(72):189-228.

3. United Nations Children's Fund, Instituto Nacional de Estadística y Censos. Situación de los niños y adolescentes en la Argentina 1990/2001. Buenos Aires: INDEC; 2003. (Serie Análisis Social $N^{\circ} 2$ ).

4. Portnoy F. El embarazo en la adolescencia y los riesgos perinatales. En: Gogna $M$, coordinadora. Embarazo y maternidad en la adolescencia: estereotipos, evidencias y propuestas para políticas públicas. Buenos Aires: CEDES; 2005.

5. Puffer R, Serrano C. Características del peso al nacer. Washington DC: OPS, OMS; 1988. (Publicación Científica No 504).

6. Moreno E. Morbimortalidad en la niñez. En: O'Donnell A, Carmuega E, coordinadores. Hoy y mañana. Salud y calidad de vida para la niñez argentina. Buenos Aires: Centro de Estudios sobre Nutrición Infantil; 1999.
7. Vinocur P. Exclusión y pobreza. Derechos y oportunidades perdidas de los niños. En: O'Donnell A, Carmuega E, coordinadores. Hoy y mañana. Salud y calidad de vida para la niñez argentina. Buenos Aires: Centro de Estudios sobre Nutrición Infantil; 1999.

8. Baranchuk NS, compilador. Medicina Siglo XXI: modelo para armar. Buenos Aires: Medicina y Sociedad Ediciones; 2001.

9. McKeown T. The Role of Medicine: Dream, Mirage or Nemesis?. Oxford: Basil Blackwell; 1979.

10. Bronfman M, Huirán R. La desigualdad social ante la muerte: clases sociales y mortalidad en la niñez. México: El Colegio de México; 1983.

11. Behm H. Desigualdad social frente a la muerte en América Latina. Santiago: CELADE; 1992. (Serie B No 96).

12. Torrado S. Estructura social de la Argentina 1945-1983. Buenos Aires: Ediciones de la Flor; 1992.

13. Wilkinson R. Unhealthy Societies. The afflictions of Inequality. Nueva York: Routledge;1997.

14. Delamónica E, Minujín A, Vandemoortele J. Crecimiento, pobreza e infancia. En: Socialis. Reflexiones Latinoamericanas sobre Política Social; vol 3. Rosario: Homo Sapiens ediciones; 2000.

15. Marmot M. Historical perspectiva: the social determinant of disease. Some blossoms 
[publicación electrónica en línea] Epidemiologic Perspectives \& Innovations 2005;2(4) [fecha de acceso 10 de octubre de 2006]. URL disponible en: http://www.epi-perspectives.com/content/2/1/4

16. Cusminsky M, Díaz Muñoz AR. La salud de los niños menores de 6 años. En Infancia y condiciones de vida. Encuesta especial para el diagnóstico y la evaluación de las metas sociales. Buenos Aires: INDEC; 1995.

17. Ramos S, Gogna M, Petracci M, Romero M, Szulik D. Los médicos ante la anticoncepción y el aborto ¿Una transición ideológica? Buenos Aires: CEDES; 2001.

18. Schwarcz R. Maternidad segura en Argentina. En: O'Donnell A, Carmuega E, coordinadores. Hoy y mañana. Salud y calidad de vida para la niñez argentina. Buenos Aires: Centro de Estudios sobre Nutrición Infantil; 1998.

19. Taucher E. La mortalidad infantil en Chile. Notas de Población. 1979;VII(20):35-72.

20. Troncoso M. Componentes sociales, económicos y culturales del proceso salud-enfermedad en el primer año de vida del niño. En: Lattes A, Farren M, MacDonald J, compiladores. Salud, enfermedad y muerte de los niños en América Latina. Buenos Aires: CLACSO, IDRC; 1989.

21. Masuy-Stroobant G. Infant Health and Infant Mortality in Europe: Lessons from the Past and Challenges for the Future. En: Corsini CA, Viazzo PP, editores. The decline of infant and child mortality. The European Experience: 1750-1990. Amsterdam: Unicef; 1997.

22. Ministerio de Salud y Acción Social. Encuesta a Población. Módulo de Utilización y Gasto en Servicios de Salud. Aglomerado Gran Buenos Aires. Situación Sanitaria de la Población. Cifras Definitivas. Buenos Aires: PRONATASS;1994. (Serie 10 NN $^{\circ} 10$ ).
23. Luppi IR, Boggie GS, Hachuel LS. Estudio de desigualdades en salud desde una perspectiva socioespacial mediante análisis de niveles múltiples. Ponencia presentada en las VI Jornadas de Debate Interdisciplinario en Salud y Población. Buenos Aires: Universidad de Buenos Aires; 2005.

24. Moura da Silva AA, Bettiol H, Barbieri MA, Sousa Riberiro V, de Farias Aragao VM, Oliveira Brito LG, Mendes Pereira M. Infant mortality and low birth weight in cities of Northeastern and Southeastern Brazil. Revista de Saúde Pública. 2003;37(6):693-698.

25. Barbieri MA, Silva A, Bettiol A, Gomes UH. Risk factors for the increasing trend in low birth weight among live births born by vaginal delivery, Brazil. Revista de Saúde Pública. 2000;34(6):596-602 .

26. D'Orsi E, Carvalho MS, Goncalves Cruz O. Similarity betwen neonatal profile and socioeconomic index: a spatial approach. Cadernos de Saúde Pública. 2005;21(5):786-794.

27. Tavares de Andrade CL, Szwarcwald CL, Granado Nogueira de Gamma S, Leal $M$. Desigualdades sócio-econômicas do baixo peso ao nacer e da mortalidade perinatal no Municipio do Rio de Janeiro, 2001. Cadernos de Saúde Pública. 2004;4(supl1):S44-51.

28. Horta BL, Barros FC, Halpern R, Victora CG. Baixo peso ao nacer em duas coortes de base populacional no sul do Brasil. Cadernos de Saúde Pública, 1996;12(supl1):S27-31.

29. Menéndez EL. Grupo doméstico y proceso salud/enfermedad/atención. Del "teoricismo" al movimiento continuo. Cuadernos Médicos Sociales. 1992;(59):3-18. 


\section{BIBLIOGRAFÍA DE CONSULTA}

Ageitos ML. Lactancia materna. En: O'donell A, Carmega E, compiladores. Hoy y mañana. Salud y calidad de vida para la niñez argentina Buenos Aires: CESNI; 1998.

Alleyne GAO. La salud en el marco de reducción de la pobreza. 5ta. Reunión del Foro Permanente para la Aplicación de la Estrategia de Cooperación al Desarrollo. Lima: OPS; 1998. (OPS/PER/99.03).
De Sarasqueta $P$, Hidalgo $S$, Siminovich $M$, Barbosa P, Gamba L, García A, Berberian G. Mortalidad postneonatal por infecciones respiratorias bajas. Antecedentes adversos de la salud y fallas del proceso de atención. Medicina Infantil.1993;1(1):10-14.

Evans RG, Hodge M, Pless IB. Si no la genética, ¿entonces qué? Vías biológicas y salud de la población. En: Evans RG, Barer ML, Marmor TR. ¿Por qué alguna gente está sana y otra no? Los determinantes de la salud de las poblaciones. Madrid: Díaz de Santos; 1996.

\section{FORMA DE CITAR}

Mazzeo V. La situación de la salud-enfermedad-atención de la primera infancia en la Ciudad de Buenos Aires entre 1990 y 2002. Salud Colectiva. 2007;3(3):285-300.

Recibido el 9 de noviembre de 2006

Versión final presentada el 16 de julio de 2007

Aprobado el 21 de agosto de 2007 